\title{
APPROACH FOR THE AUTOMATED AND DATA-BASED DESIGN OF MECHANICAL JOINTS.
}

\author{
Zirngibl, Christoph; \\ Schleich, Benjamin; \\ Wartzack, Sandro \\ Friedrich-Alexander-Universität Erlangen-Nürnberg
}

\begin{abstract}
As a result of the increasing challenges in the field of lightweight constructions, the demand for efficient joining technologies is continuously rising. For this purpose, cold forming processes offer an environmental friendly and fast alternative to established joining methods (e.g. welding). However, to ensure a high reliability, not only the selection of an appropriate procedure, but also the dimensioning of the individual joint is essential. While product designers can rely on a wide range of design principles for thermal processes, the dimensioning and evaluation of mechanical joining processes is mainly based on expert knowledge and a few experimental tests. Although few studies already investigated the numerical analysis of mechanical joints, an approach for the sustainable and consistent optimization of the strength and reliability of joining connections for varying use-cases is not available yet. Motivated by this lack, this paper presents an approach for the automated transfer of information within the process chain and the data-based analysis of mechanical joints by using clinching as an example. Therefore, the CRISP-DM reference model is used for the systematic data mining.
\end{abstract}

Keywords: Lightweight design, Optimisation, Machine learning, Clinching, CRISP-DM

\author{
Contact: \\ Zirngibl, Christoph \\ Friedrich-Alexander-Universität Erlangen-Nürnberg \\ Engineering Design, KTmfk \\ Germany \\ zirngibl@mfk.fau.de
}

Cite this article: Zirngibl, C., Schleich, B., Wartzack, S. (2021) 'Approach for the Automated and Data-Based Design of Mechanical Joints.', in Proceedings of the International Conference on Engineering Design (ICED21), Gothenburg, Sweden, 16-20 August 2021. DOI:10.1017/pds.2021.52 


\section{INTRODUCTION}

Given the increasing number of mixed metal connections and coated components in lightweight design, the application of mechanical joining processes provides a robust and efficient solution of connecting two or more metal sheets by local cold forming. Due to improving production standards and the awareness of the joining reliability, product developers not only focus on the selection of a suitable process, but also on the dimensioning and design of optimal joints. However, in comparison to established thermal joining methods like welding, only a small number of design and formation principles are available for mechanical joining processes (Drossel et al., 2013).

Consequently, the gain of knowledge and the modification of process parameters mainly require high cost- and time-intensive development iterations. Due to the increasing complexity of multidimensional distributions, experimental studies are only applicable for a limited number of parameter variations. Nevertheless, to enable the identification of optimal joint connections, the application of parametric studies in combination with data-based methods already showed high potentials for the analysis of a wide range of different tool geometries (Zirngibl et al., 2020). However, machine learning methods like artificial neural networks (ANN) often require a sufficiently high amount of data. In order to achieve an adequate balance between a lowest possible data sampling effort and the usability of data-based techniques, parametric studies often base on statistical methods for the efficient design of experiment.

In this context, the numerical analysis of clinch joints is already investigated in few papers. Mainly, the aim is to improve the strength of a clinch joint by using statistical sampling techniques combined with machine learning methods. For instance, Oudjene et al. (2009) use the response surface methodology (RSM) for the shape optimization of clinching tools (punch, die). For this purpose, a Moving LeastSquare approximation obtains accurate results and enhance the resistance of clinch joints to tensile loading. Based on a central composite DoE with three levels, numerical FE simulations generate a database for the following derivation of response surfaces. In the work of Lambiase et al. (2013) an artificial neural network is trained to improve the clinch joints' strength for the use of extensible dies. Therefore, a finite element model provides the basis to vary different clinch tool configurations. Based on this, the ANN is trained and validated by predicting the impact of tool geometries (punch, die) on target features (e.g. interlock thickness). Following, a genetic algorithm (GA) offers the opportunity to obtain optimized tool configurations. Drossel et al. (2013) focuses on the implementation of FE simulations combined with the intelligent design of experiment (Latin hypercube design). Resulting, robustness and sensitivity analysis present an adequate way to determine the effect of varying parameter combinations on the joining results. The identification of the relevant process and tool parameters supports the following generation of a well-balanced tool geometry by using an adaptive response surface method. Wang et al. (2017) describe an approach that also takes process parameters like blank holder force and punch speed into consideration. Based on the result of a sensitivity analysis, a reduced design matrix (Box-Behnken design) of nine parameters enables the following approximation of regression models. For this, the RSM provides the opportunity to identify deep relations between independent (e.g. punch diameter) and dependent factors (e.g. interlock thickness). Finally, the application of a genetic algorithm (NSGA-II) allows a multi-objective optimization to determine parameter combinations for the simultaneous maximization of the neck and interlock thickness.

In summary, the papers show approaches for the identification of improved clinch joints. While only a small number of materials or sheet combinations are analysed, product designers often need to manually adjust the parametric studies and restart the optimization process for changing conditions (e.g. modification of parameter ranges or selecting a different material combination). This can lead to time- and cost-intensive analysis iterations. Also, media breaks (e.g. between DoE database and FE system) or the manual evaluation of target values can cause a loss or misinterpretation of information. Thus, the analysis quality is strongly dependent on the consistent transfer of information between each workflow step. Furthermore, to obtain optimal tool geometries, the use of meta-model assisted genetic algorithms enable a fast and extensive method for the identification of possible parameter combinations. However, since the accuracy of the results is strongly dependent on the selection of predictable machine learning techniques, the comparison of different methods is crucial.

Although few studies already investigated the numerical analysis of mechanical joints, an approach for the automated and consistent optimization of geometrical clinch joint characteristics in versatile process chains is not available yet. Motivated by this lack, this paper presents an approach for the automated transfer of information within the process chain and the data-based analysis and 
improvement of mechanical joints. For this purpose, clinching is used as an example and provides the basis for a later discussion on whether the approach is applicable and transferable to other mechanical joining processes. In addition, the creation of a solution space based on a meta-model assisted genetic algorithm provides the opportunity to select the best fitting design for varying process requirements. Thereby, the implementation of an interface to a CAD software enables the product developers to directly realise a CAD representation of the chosen tool designs. In addition, the approach offers the opportunity to incorporate manufacturing knowledge by adding individual optimization boundaries. To guarantee a systematic application of statistical methods for the discovery of knowledge in databases, the cross industry standard process for data mining (CRISP-DM) is used.

\section{THEORETICAL BACKGROUND}

\subsection{Clinching Process}

According to Fahrenwaldt and Bye $(2006,2014)$ clinching is a mechanical joining process that provides the opportunity to join profiles or metal sheet entirely based on local cold forming. For this purpose, the creation of a force- and form-fitting connection requires no auxiliary joining elements like rivets. Additionally, clinching offers a cost-effective and environmentally friendly procedure for the use in lightweight design. This includes the variation of dissimilar sheet thicknesses or the joining of less weldable materials. Consequently, clinching has become an important mechanical joining process in the automotive (e.g. car body and frame components) and appliance industry. To achieve a sufficiently high strength of the sheet connection, it is necessary to form geometrical characteristics (e.g. a specific neck and interlock thickness). Therefore, the sheets are positioned between the blank holder and die. Subsequently, the moving of the punch initialises a compression and deformation (spreading, compression and extrusion) within the materials. Following, the filling of the die starts and the forming of the final joint geometry begins. Figure 1 shows geometrical characteristics of a clinch joint.

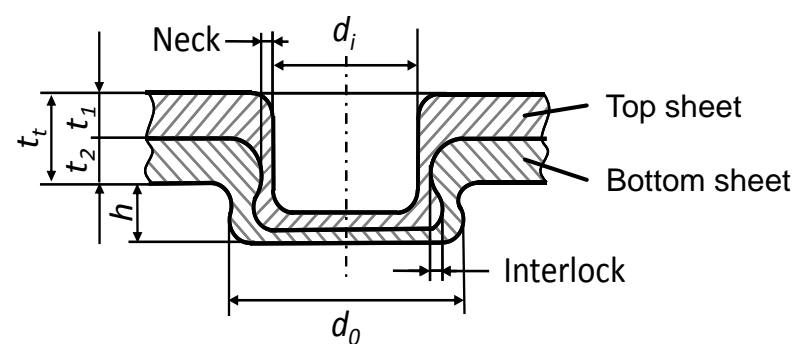

Figure 1. Geometrical characteristics of a clinch joint according to DVS (2009)

\subsection{CRISP-DM}

Based on Witten et al. (2017), data mining can be described as the process that identifies patterns and meaningful correlations in data and supports the accurate and efficient extraction of knowledge. In this context, the CRISP-DM reference model represents a systematic approach for the successful implementation and performance of data mining projects. At the beginning, the product designers need to define what information and data are necessary to achieve the particular goals. Afterwards, the raw data have to be cleaned and prepared for the following performance of machine learning algorithm. This includes for example the standardization/normalization or the removing/filling of missing information.

In the next step, the selection and performance of several modeling techniques and the calibration and optimization of their estimation parameters enable the creation of a structural description of information in the database. Subsequently, to ensure the achieving of the defined business objectives, it is necessary to evaluate the constructed models. For this, a few measures like the root-mean-square error (RMSE) and the coefficient of prognosis (CoP) offer the opportunity to determine the performance of a machine learning method by calculating the difference between the predicted and the observed values. Finally, the deployment of the evaluated model includes the implementation in the practice. This often involves the presentation of the achieved results and the integration into a software system. To ensure a systematic application of statistical methods for the following multi-objective optimization of the clinch joint, this paper is mainly focusing on the data preparation, modeling and evaluation steps. 


\section{GENERAL APPROACH}

Following, an overview of the presented approach and the explanation of the individual workflow steps is provided in more detail. Consequently, the research question of how the automated and consistent transfer of information within the process chain ensure the data- and knowledge-based improving of clinch joints for varying use-cases shall be answered. Therefore, the systematic generation of a database by using intersections between the design of experiment and the CAD-/FEsoftware is described in the beginning. Subsequently, the pre-processing of the generated raw data provides the basis for the application of several data mining methods. Finally, the use of different regression measures evaluates the performance of the developed estimators. These steps are described in sequence, starting with the generation of the database. Figure 2 shows an overview of the general approach including the sampling of data following by the application of CRISP-DM sub-steps and the final optimization processing.

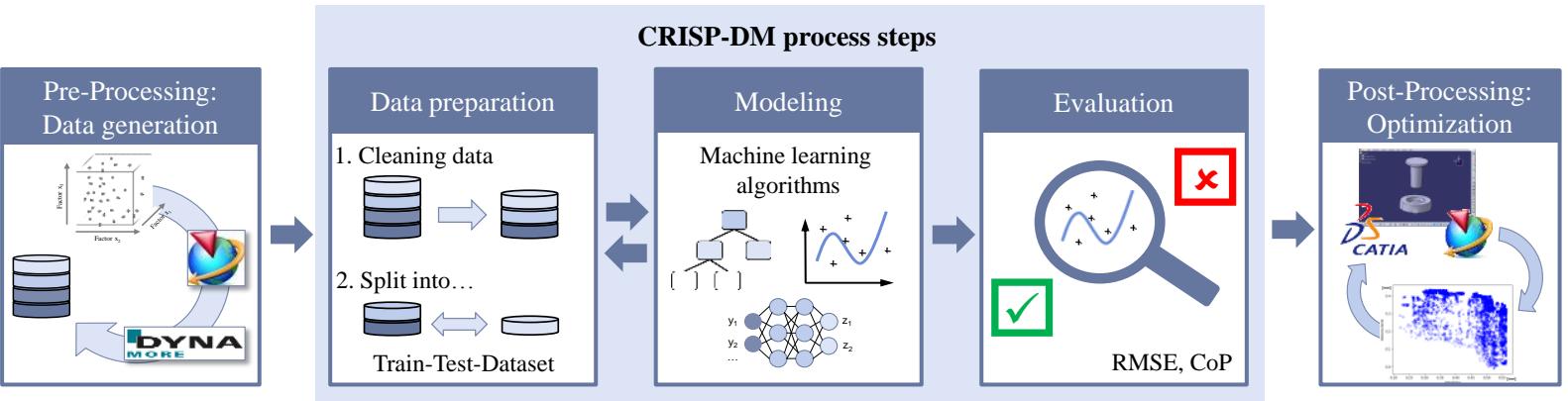

Figure 2. Overview of the general approach

\subsection{Pre-Processing: Data generation}

To ensure an efficient and accurate application of several machine learning methods, it is necessary to provide a sufficiently large amount of data. However, due to media breaks between the data mining steps and the often manually-based evaluation of the feature variables, information can be lost or misinterpreted. The subsequent decrease of the data quality can lead to poor performances of the following modeling and optimization procedures. To counteract them, the presented approach includes the implementation of a graphical user interface (APAOS, Automated Parameter Analysis and Optimization System) to secure the consistent and efficient transfer of information within the process chain and the automatic execution of individual analysis steps. Therefore, product developers have the opportunity to build up a design of experiment (DoE) based on individual factor spaces. For this purpose, it is possible to vary geometrical (e.g. tool dimensions), process (e.g. punch speed) and material (e.g. flow curves) parameters that describe the investigated clinch joining process.

According to Siebertz et al. (2017), the use of a Latin hypercube design ensures the generation of a near-randomly sampling set and the avoidance of artificial correlations between the factors by defining a sufficiently large amount of data points per parameter. Following, the generated DoE provides the basis for the subsequent sampling process.

In this context, the challenge is to guarantee the consistent transfer of information within the individual sampling steps. Therefore, the particular intersections between the domains (e.g. DoE, CAD, FEM) and the generation of a backend (database) allow an automated and efficient exchange of data. This also ensures that no information is lost due to domain-dependent media breaks within the process chain.

As a first step, an algorithm loads the generated DoE data from the backend and starts the sampling of CAD geometries (e.g. die and punch). Therefore, a row-by-row query identifies the related geometrical factors and enables the following consistent data transfers to a parameterised CAD-model. Afterwards, the created parts provide the geometrical frame conditions for the subsequent building of FE simulations. For this, the use of an experimentally validated 2D-axisymmetric FE model (LS-DYNA) for the clinching of sheet metals secure the reliability and accuracy of the following simulation steps

In the next step, an algorithm performs the automated import and meshing of the particular tool geometries and the adjustment of varying process and/or material parameters. After the completion of the previous steps, the automated execution of the numeric clinching processes is performed. This step can last several days, depending on the available CPU performance and the amount of data samples. Subsequently, a database is obtained which contains a large number of different numerically generated 
clinch connections. For the following analysis steps, the removing of irrelevant geometry parts (e.g. die, blank holder and punch) assists the sufficient identification of the required clinch joint geometrical characteristics (neck and interlock thickness) and the prevention of taking unimportant information into consideration. Afterwards, an algorithm interpolates (B-Splines) between the contour outliner nodes of the remaining joined material sheets and transfers all related geometrical data (e.g. x-y node coordinates) into an array. Based on this, an unsupervised machine learning method (clustering algorithm) enables the classification of the dataset into a die-side, an inner and a punch-side contour. Hence, an agglomerative algorithm merges the geometrical data by their minimal increase to a particular linkage distance. Resulting, the defined inner and die-side contour build the basis for the following identification of the interlock and neck thickness. Hereby, it is possible to determine turning points of the numerical clinch joints by monitoring the $\mathrm{x}$-coordinates of the inner contour. In this case, an algorithm iterates through the database to identify changes of mathematical signs that indicates the occurrence of potential turning points. Then, the horizontal distance between the determined turning points provides the accurate identification of the interlock thickness. To deviate the neck thickness, an algorithm builds a perpendiculars from the punch-side to the inner contour of the investigated clinch joint. Following, the shortest perpendicular distance between the contours represents the required target feature. As a summary, figure 3 shows the several steps for the automated generation of a database.

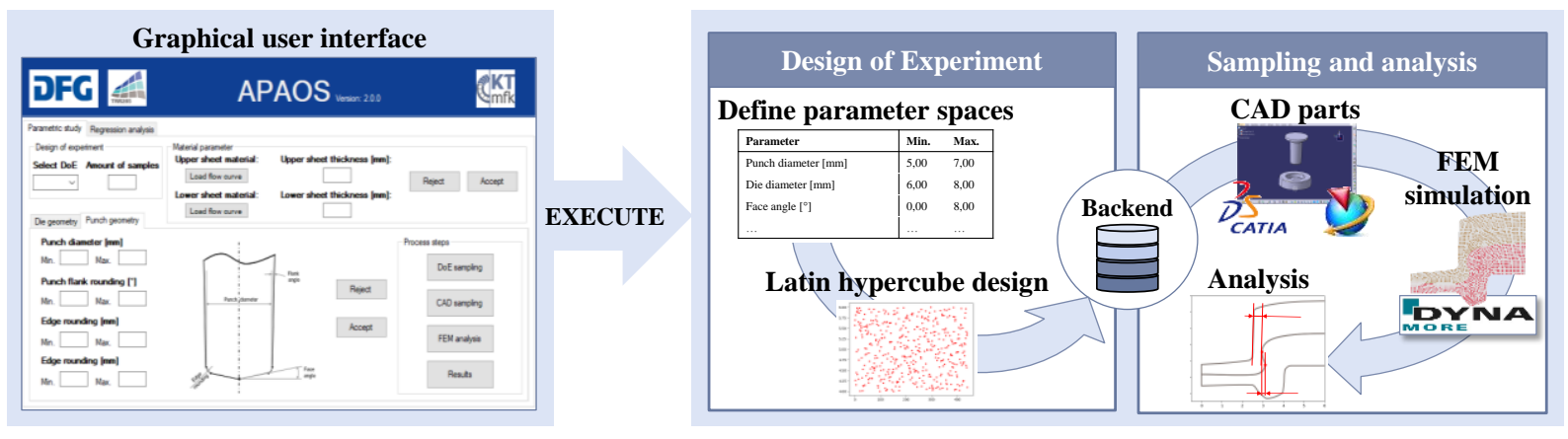

Figure 3. Overview of the GUI and the steps for the automated generation of a database

\subsection{Data preparation}

The generated database often includes inaccurate and/or non-standardised values. Due to the decreasing performance of machine learning methods on poor-quality data, it is generally well worth the effort to have a deeper focus on cleaning up the given dataset. This mainly involves the identification of missing, irrelevant or noisy samples. For this purpose, it is important to find the balance between the reduction of samples and the guarantee of a still sufficiently high quantity of data.
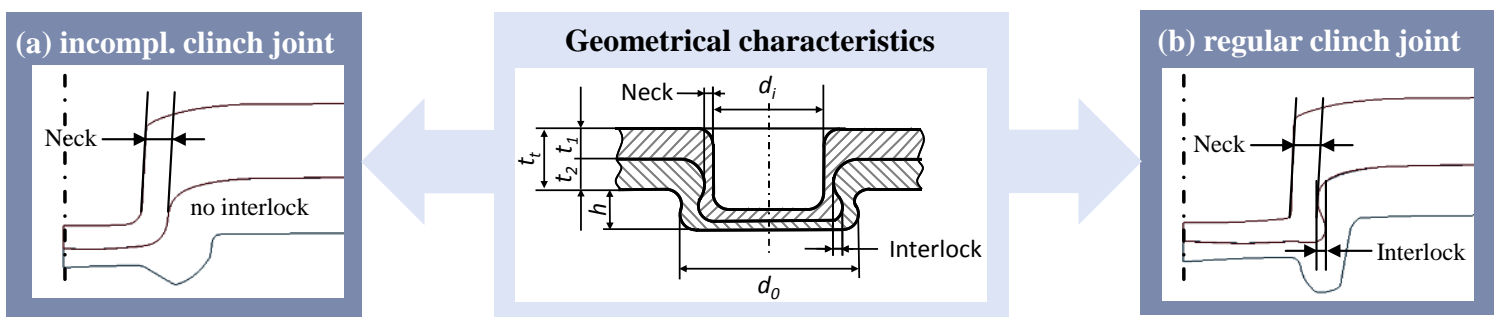

Figure 4. Example of an incomplete and fully created clinch joint

Therefore, the definition of selection criteria is necessary in advance. This includes the evaluation of the applied FE-simulations and the consideration of the given numerical feature scales. As presented before, the forming of a clinch joint indicates the specification of several geometrical characteristics (e.g. interlock thickness). The insufficient generation of these leads to an incorrect joining of the metal sheets. Figure 4 shows an example of an incomplete (a) and fully (b) created clinch joint.

Thus, the erroneous simulation results have to be removed from the created database. This can be achieved by iterating through the dataset and deleting each line that includes an interlock value of zero. Furthermore, the inaccurate forming of joint outliner contours caused by error terminated simulations can lead to a misinterpretation of underlying patterns in the data and have to be removed as well. Instead of preparing the database manually, the product designers can rely on an algorithm that automatically detects incorrect or irrelevant features. Since machine learning methods often perform less effective on 
different numerical attribute scales, the application of feature scaling algorithms enable the transformation of all attributes to an equal scale level. As a result, outliner or noise within the dataset have a lower impact on the identification and fitting of a suitable machine learning model.

\subsection{Modeling}

According to Witten et al. (2017) the modeling step represents the core of machine learning processes and builds the basis for the creation of a structural description of information in the database. Therefore, the selection and performing of several modeling techniques and the calibration and optimization of their estimation parameters enable the identification of best solutions for complex problems. In Géron (2019) the field of machine learning methods is divided in different categories according to the required type and amount of supervision. This includes the following classes: supervised, unsupervised, semisupervised and reinforcement learning. Due to the previous determination of the target features (labels) and the following integration into the training set, the presented approach belongs to the supervised learning tasks. Subsequently, the application of regression algorithms (e.g. polynomial regression models and artificial neural networks) offer the opportunity to estimate numeric values on an unknown dataset. Following, the identification of the best performing technique secures an individual model selection for different estimation tasks.

\subsection{Evaluation}

This step aims to get a deeper understanding of the estimators' performance. That means, how satisfying a machine learning technique generalizes to an unknown set of data. Therefore, the comparison of different performances ensure the identification of the best performing technique for the pattern recognition and the identification of information within the database. To avoid overfitting, the use of a 10 -fold cross validation enables the numerical evaluation of the predictive models by splitting the database into several training and test sets. According to Witten (2017) few measures are suitable for the evaluation of a numeric predictions' success. For example, the root means-squared error (RMSE) defined by Mayer et al. (1993) is one of the most commonly and principal used techniques. Due to its calculation of the difference between the predicted and the observed values, low RMSE values indicate a well performing estimator. Also, concerning to the work of Most et al. (2008), the coefficient of prognosis (CoP) offers a sufficiently accurate independent measurement by using only points that are not applied for the building of the approximation model. As higher the value is (max 1.0), the better the machine learning technique performs.

\subsection{Post-Processing: Generation of optimized clinch tool geometries}

To get a sufficiently high strength of the investigated clinch joint, the aim is to simultaneously maximize the interlock and neck thickness. Due to the interdependence of the target features, the represented use case is defined as a multi-objective optimization problem. This means, that at a specific optimization step, the improving of one variable results in the worsening of the other objective functions. For that, Coello (2018) describes a wide range of optimization methods that offer opportunities to identify the best trade-off between the competing variables. Especially, the concept of Pareto optimizations show a systematic procedure to find a set of optimal solutions that are superior to additional solutions in the defined parameter space but non-dominated to each other. For this purpose, evolutionary algorithms offer well-performing methods to deal with a large field of optimization problems.

In the presented approach the use of the non-dominated sorting genetic algorithm II (NSGA-II) enables the efficient creation of Pareto frontiers by generating offsprings based on the comparison of crowding distances. To ensure accurate results, it is recommended to apply constraints on the multiobjective optimization process. This includes the previous determined parameter spaces and the definition of manufacturing boundaries (e.g. minimal interlock and neck thicknesses). In addition, to guarantee the realization of the identified geometries, the selection of manufacturing tolerances are represented in the genetic algorithm. Therefore, the step size for the creation of individuals (set of parameters) is adapted to an accuracy of $+/-0.01 \mathrm{~mm}$.

Due to the implementation of an intersection between the resulting Pareto frontier and the CAD systems, product designers can then automatically derive a CAD model for the chosen geometrical clinch tool parameter values by selecting a solution point in the appearing plot. Finally, to evaluate the recent tool geometry and process parameters, a numeric FE simulation is carried out and the results are compared to the initial clinch joint. 


\section{RESULT AND DISCUSSION}

For the automated generation and performance of a parametric study, involving the analysis and optimization of mechanical joints, the presented approach is demonstrated using clinching of two similar metal sheets $(2.0 \mathrm{~mm} / 2.0 \mathrm{~mm}$; EN-AW-6014) as an example. Therefore, the generation of the required database was carried out at the beginning. For this, the implemented GUI enables the definition of particular parameter spaces and the loading of the material flow curves. Thus, the variation of ten clinch tool geometry parameters (e.g. punch and die diameter) and two process parameters (punch speed and bottom thickness) offer the opportunity to investigate a large range of possible clinch joints for the given scenario. Table 1 gives an overview of the defined parameter spaces and thus builds the basis for the following approach demonstration.

Table 1. Overview of defined parameter spaces

\begin{tabular}{|c|c|c|c|c|c|c|c|c|c|c|c|c|}
\hline & \multicolumn{12}{|c|}{ Input parameter for the clinching of $2 \mathrm{~mm} / 2 \mathrm{~mm} \mathrm{EN}-\mathrm{AW}$ - 6014 sheet metals } \\
\hline & \multicolumn{4}{|l|}{ Punch } & \multicolumn{6}{|l|}{ Die } & \multicolumn{2}{|l|}{ Process } \\
\hline & $\begin{array}{l}\text { Punch } \\
\text { diameter }\end{array}$ & $\begin{array}{l}\text { Corner } \\
\text { radius }\end{array}$ & $\begin{array}{l}\text { Side draft } \\
\text { angle }\end{array}$ & $\begin{array}{l}\text { Face draft } \\
\text { angle }\end{array}$ & $\begin{array}{l}\text { Die } \\
\text { diameter }\end{array}$ & $\begin{array}{l}\text { Die } \\
\text { depth }\end{array}$ & $\begin{array}{l}\text { Groove } \\
\text { depth }\end{array}$ & $\begin{array}{l}\text { Bottom } \\
\text { diameter }\end{array}$ & $\begin{array}{l}\text { Groove } \\
\text { diameter }\end{array}$ & $\begin{array}{l}\text { Draft } \\
\text { angle }\end{array}$ & $\begin{array}{l}\text { Punch } \\
\text { speed }\end{array}$ & $\begin{array}{l}\text { Bottom } \\
\text { thickness }\end{array}$ \\
\hline Min. & $4.5 \mathrm{~mm}$ & $0.1 \mathrm{~mm}$ & $0.0^{\circ}$ & $3.0^{\circ}$ & $7.5 \mathrm{~mm}$ & $0.8 \mathrm{~mm}$ & $0.5 \mathrm{~mm}$ & $3.5 \mathrm{~mm}$ & $5.4 \mathrm{~mm}$ & $0.0^{\circ}$ & $2.0 \mathrm{~mm} / \mathrm{s}$ & $0.6 \mathrm{~mm}$ \\
\hline Max. & $6.0 \mathrm{~mm}$ & $0.6 \mathrm{~mm}$ & $4.0^{\circ}$ & $8.0^{\circ}$ & $8.5 \mathrm{~mm}$ & $1.8 \mathrm{~mm}$ & $1.3 \mathrm{~mm}$ & $5.0 \mathrm{~mm}$ & $7.0 \mathrm{~mm}$ & $8.0^{\circ}$ & (const.) & (const.) \\
\hline
\end{tabular}

Based on this, the creation of a suitable design of experiment (DoE) ensures the sampling for multidimensional parameter distributions. Consequently, a DoE database is generated including 300 samples, which means a resolution of 30 points per varied parameter.

In the next step, an algorithm performs the sampling of the CAD-geometries and the generation of FEmodels by automatically loading the relevant data to a connected CAD-software (SIEMENS NX or CATIA V5). Therefore, the implemented intersections between the required software and the backend ensure a consistent and continuous information flow without the risk of data loss. Thus, it is possible to quickly adapt the parameterised CAD model and to create various clinch tool geometries in a short time. In the next step, the generation of the FE simulations is achieved by loading the CAD parts and updating the dummy model according to the particular parameters. For the final execution of the FE models, the GUI offers the opportunity to enter available CPU-cores and calculates the estimated termination date.

Once the simulation steps are done, an algorithm iterates over the generated clinch joints and automatically determines the geometrical characteristics (neck and interlock thickness). As a result of the pre-processing steps, 282 evaluated simulations build the database for the following modeling procedure. Thus, 18 simulations $(6 \%)$ showed error terminations or design failures and were removed from the database. Subsequently, the automated performance of different machine learning methods and the application of the 10-fold cross validation ensure the pattern recognition and the identification of information in the database. Therefore, an algorithm randomly splits the database into 10 equal-sized datasets (folds). Then, the estimators are trained several times by selecting 9 folds as the training data and measuring the performance by using the remaining fold for the evaluation data set. In summary, the cross-validation allows to get a deeper understanding of how each predictor performs, but also an overview of how precise these predictions are by calculating the standard deviation. Figure 5 shows the machine learning performances $(\mathrm{CoP})$ for the estimation of the interlock and neck thickness.
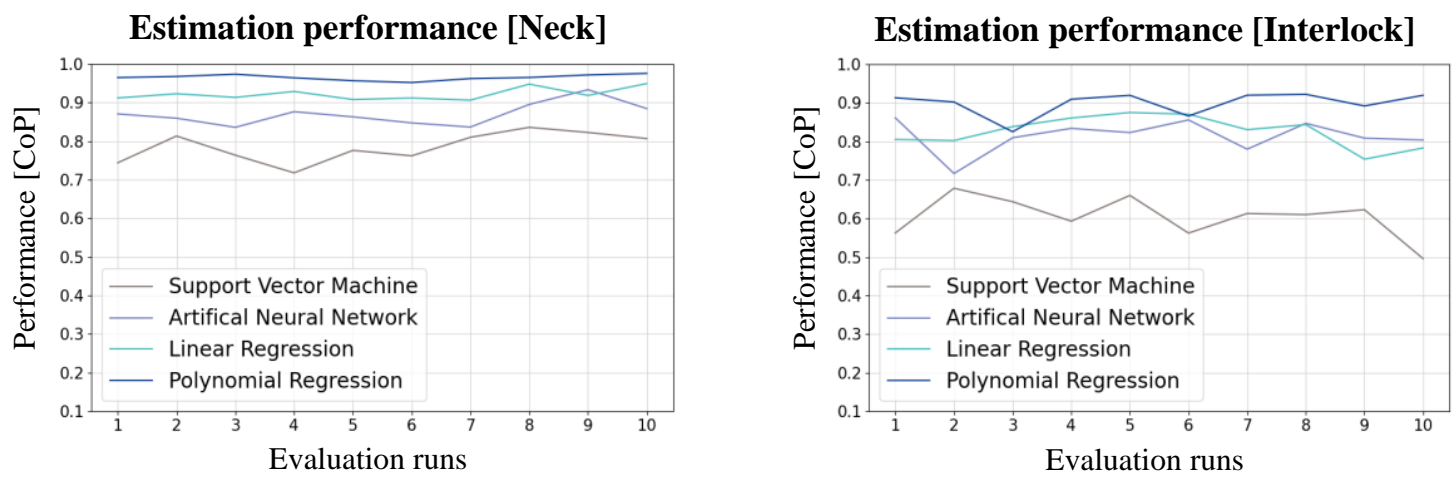

Figure 5. Performance of different machine learning methods for the interlock thickness

One can see, that the techniques perform quite different for the varying subsample data. While the estimation of the neck thickness achieves continuously high and precise CoP values, the prediction of 
the interlock thickness shows strong uncertainties for several evaluation runs. In summary, the linear and polynomial regression model reach satisfying results for the prediction of both target features.

Based on this, the selection of the best performing machine learning methods builds the basis for the following sensitivity analysis. Therefore, an algorithm automatically stores and serializes the chosen object to the backend and starts the mathematical analytical process. Thus, the sensitivity analysis offers a deeper understanding of the relationships between the input and target features. Those results will be stored in the backend and lead to an enlargement of the empirical dataset and the improving of the following suggestions of default values for the initial parameter spaces.

In the next step, an algorithm starts the multi-objective optimization process. As already described by Sauer et al. (2020), the automated generation of a solution space by using a meta-model assisted GA shows vast potentials for the identification and evaluation of different part designs in the field of metal forming. Hereby, it is possible to realize a higher amount of generation runs and populations (set of individuals) in comparison to the direct communication between a GA and the parametric FE-model by requiring significantly less computational and time resources. This allows to create many design alternatives and the formation of a solution space with a single optimization run. Furthermore, based on the greater amount of design point, it is also possible to evaluate whether the solution space shows global optima (Pareto front) or only local maxima. However, to guarantee a high reliability of the results, it is necessary to identify best fitting machine learning methods in advance.
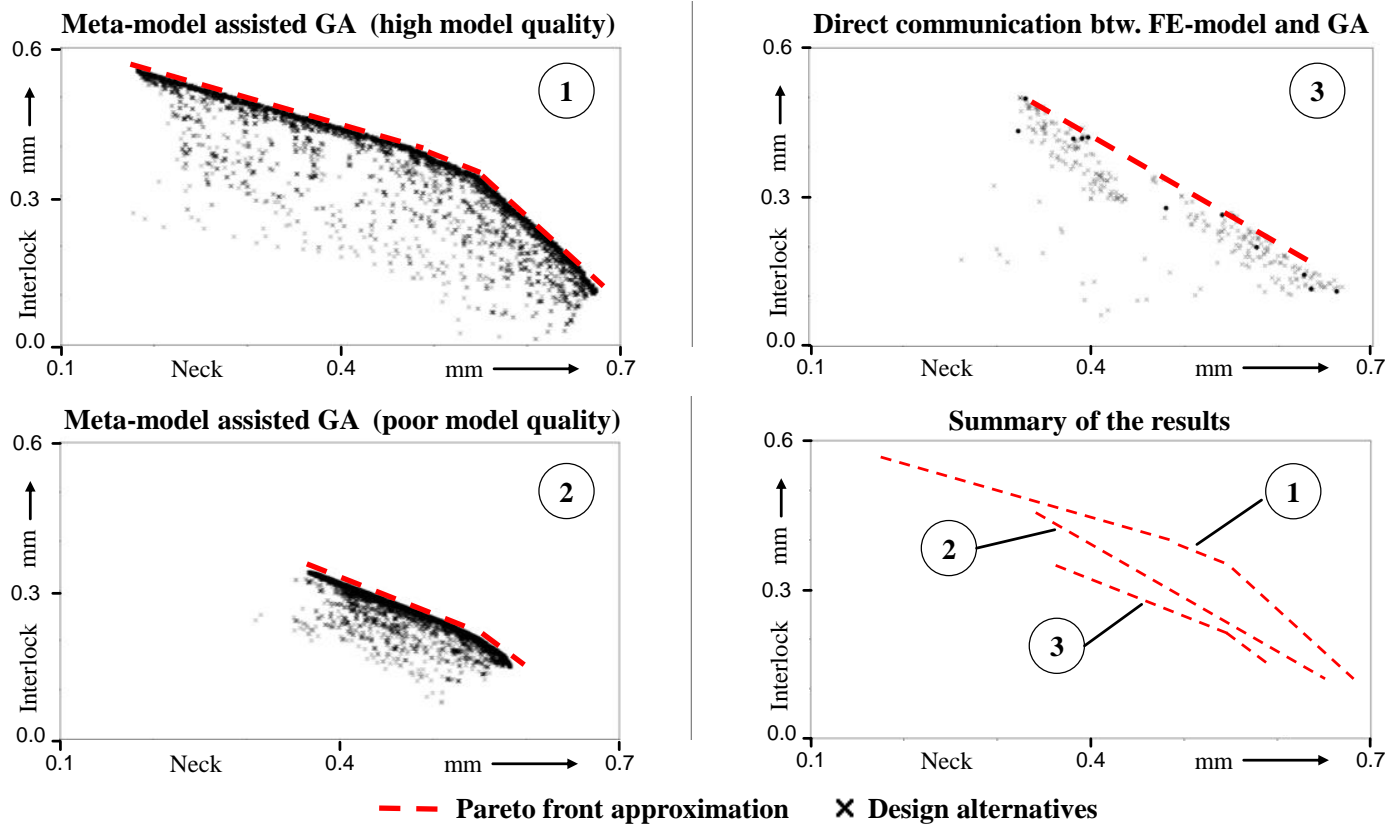

Figure 6: Solution spaces for different applied genetic algorithms.

Thereby, poor-quality models can lead to an incorrect formation of design points and thus to a false interpretation of the created solution space. As an example, figure 6 shows an overview of different Pareto frontiers. At this, the solution space of the directly communicating GA bases on a population size of 12 and a number of generations of 25. This represents the same amount of simulation runs in comparison to the initial database for the building of meta-models.

As already mentioned, the application of a NSGA-II optimization algorithm offers the opportunity to generate a design solution space by maximizing both the neck and interlock thickness. To implement individual demands of product developer, it is possible to incorporate manufacturing knowledge into the solution space by adding external optimization boundaries. For the represented case study, the minimum interlock thickness is defined by $0.1 \mathrm{~mm}$ or higher, while the minimum neck thickness should not undercut a value of $0.3 \mathrm{~mm}$.

Afterwards, the intersection between the CAD software and the graphical representation of the solutions transfers the defined geometry parameters to the parametrized CAD model by selecting a particular point on the Pareto frontier. Through this, the approach offers the opportunity to select the best fitting solution for individual and changing joint requirements and the automatically creation of the relating tool configurations. Figure 7 shows a solution space including manufacturing boundaries and resulting clinch joints for different design alternatives. 


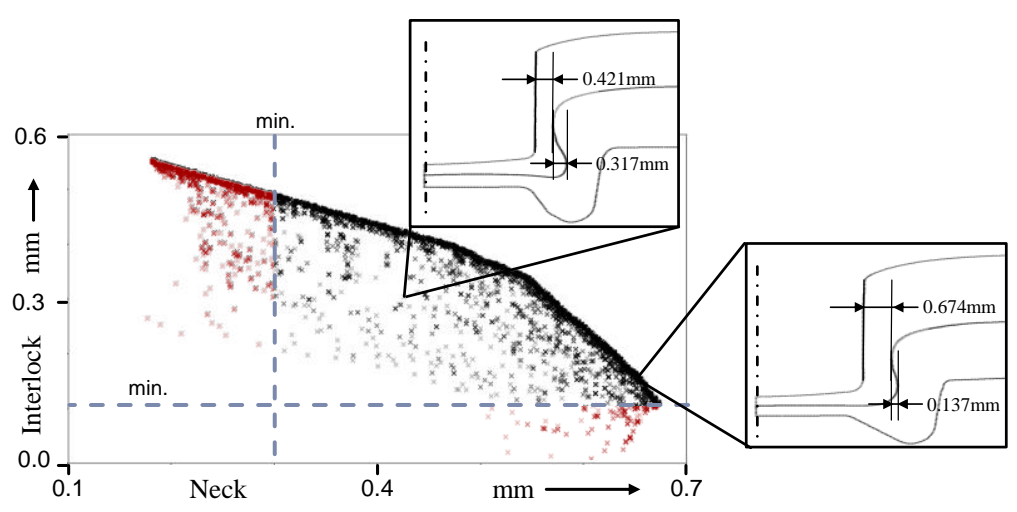

Figure 7: Solution space and resulting clinch joints for different design alternatives

In summary, the presented approach for the automated and data-based analysis of mechanical joints show an efficient alternative in comparison to the manual design and performance of optimization studies. However, the exact and robust extraction of improved clinch joints is strongly dependent on the chosen parameter. For example, product designers can only rely on a limited and previously implemented number of parameters that are available via the GUI. Also, the accurate selection of minimal and maximal parameter spaces is necessary to achieve significant results. Thus, wrongly or inaccurate values can lead to an insufficient analysis of the clinch joint. To prevent this, the implementation of default values based on previous investigated frame conditions (e.g. similar sheet or metal combinations) is recommended. These values are continuously improved by each parametric study performance and the collection of the generated empirical data. Furthermore, the integration of manufacturing knowledge that includes design restrictions and rules can support the product designers by choosing suitable parameter spaces. For the design of joining connections, product designers often require a deeper understanding of the joint strength for different loads (e.g. shear and tensile load). Therefore, the implementation of evaluated tensile tests for the 2D-axisymmetric FE model can lead to an enlargement of the approaches' applicability. Also, the results of the optimized tool geometries for the given scenario are dependent on the following production accuracy. For example, the randomly variation of tool geometries based on the realizable production tolerances can have a strong effect on the actual achievable results (Drossel et al., 2013). Consequently, to clarify the impact of varying parameter on the created clinch joints, it is recommended to implement robust analysis.

\section{CONCLUSION}

In the context of the numerical design and optimization of mechanical joining techniques, the presented paper shows an approach for the automated and data-based analysis of mechanical joints by using clinching as an example. Therefore, the approach introduces opportunities for the consistent and efficient transfer of information within the process chain and the automated execution of individual analysis steps. The application of machine learning and meta-model assisted multi-objective optimization algorithms enable the following identification of design solution spaces for varying clinch tool geometry and process parameters. Based on this, the product developers can select the best fitting solution for desired joint requirements. For the accurate definition of initial parameter spaces, it is recommended to implement default values as a support for the product developers based on existing production constraints and the gathered empirical information of previous studies. Thus, the approach enables the reuse of data and the building of a knowledge database for the efficient analysis of mechanical joining processes. Although, the manual and/or separate design and dimensioning of mechanical joints is the most established method, the presented contribution shows a valuable approach for the adaptable and automated performance of parameter studies including a genetic algorithm providing design optima. Also, the use of clinching as an example demonstrated high potentials for the application in the field of mechanical joining process. In order to evaluate this statement, the implementation of further joining techniques like riveting will be realized in the future. Thus this approach paves the way to the data-driven design of mechanical joining processes in the context of versatile process chains. 


\section{ACKNOWLEDGMENTS}

Funded by the Deutsche Forschungsgemeinschaft (DFG, German Research Foundation) - TRR 285 B05 - Project-ID 418701707

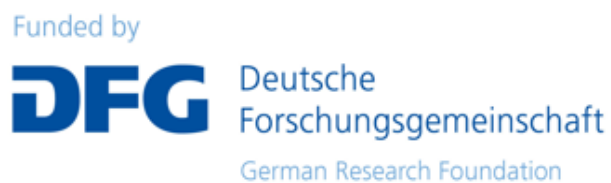

\section{REFERENCES}

Bye, C., Timmermann, R., Steber, M. (2014), "Mechanisches Fügen von Dünnblechen“, In: Feldmann, K., Schöppner, V., Spur, G. (Ed.), Handbuch Fügen, Handhaben und Montieren, Carl Hanser Verlag, München.

Coello, C.A.C. (2018), “Multi-objective Optimization“, In: Martí R., Pardalos P., Resende M. (Ed.), Handbook of Heuristics, Springer, Cham. https://doi.org/10.1007/978-3-319-07124-4_17.

DVS (2009), "Clinchen - Überblick“, In: DVS-Fachbücher Vol. 184 (Ed.), Taschenbuch DVS-Merkblätter und Richtlinien Mechanisches Fügen, DVS Media, Düsseldorf.

Drossel, W.G., Israel, M. (2013), "Sensitivitätsanalyse und Robustheitsbewertung beim mechanischen Fügen“, EFB-Forschungsbericht Nr. 323, EFB e.V., Hannover.

Eshtayeh, M., Hrairi, M. (2016), "Multi objective optimization of clinching joints quality using Grey-based Taguchi method", The International Journal of Advanced Manufacturing Technology, Vol. 87, pp. 233-249. https://doi.org/10.1007/s00170-016-8471-1.

Fahrenwaldt, H.J. (2006), "Fügen durch Umformen“, In: Fahrenwaldt, H.J., Schuler, V., Twrdek, J. (Ed.), Praxiswissen Schweißtechnik. Vieweg. https://doi.org/10.1007/978-3-8348-9192-1_6.

Géron, A. (2019), Hands-on Machine Learning with Scikit-Learn, Keras, and TensorFlow, O'Reilly UK Ltd., Sebastopol.

Lambiase, F., Di Ilio, A. (2013), "Optimization of the clinching tools by means of integrated FE modeling and artifical intelligence techniques”, 8th CIRP Conference on Intelligent Computation in Manufacturing Engineering, Ischia, 18-20 July 2012, Elsevier B.V., pp. 163-168. https://doi.org/10.1016/j.procir.2013.09.029.

Mayer, DG., Butler, DG. (1993), "Statistical validation. Ecological Modelling", Amsterdam, Elsevier Science Publishers B.V., Vol. 68, 21-32, https://doi.org/10.1016/0304-3800(93)90105-2

Most, T., Will, J. (2008), "Meta-model of Optimal Prognosis - An automatic approach for variable reduction and optimal metamodel selection", Proceedings of the Weimar Optimization and Stochastic Days 5.0, Weimar (Germany), 20.-21.11.2008, https://doi.org/10.13140/2.1.2194.4007.

Neugebauer, R., Riedel, F. and Marx, R. (2010), “Entwicklung eines Konstruktionssystems für den rechnerischen Festigkeitsnachweis von punktförmig mechanisch gefügten Bauteilen”, $E F B$ Forschungsbericht Nr. 376, EFB e.V., Hannover.

Oudjene, M., Ben-Ayed, L., Batoz, J-L. (2009), "Shape optimization of clinching tools using the response surface methodology with Moving Least-Square approximation”, Journal of materials processing technology, Vol. 209, pp. 289-296. http://doi.org/10.1016/j.jmatprotec.2008.02.030.

Sauer, C., Schleich, B., Wartzack, S. (2020), "Meta-model based generation of solution spaces in sheet-bulk metal forming". Proceeding of 30th CIRP Design, http://doi.org/10.1016/j.procir.2020.02.247

Siebertz, K., van Bebber, D., Hochkirchen, T. (2017), Statistische Versuchsplanung. Design of Experiments $(D o E)$, Springer Vieweg, Wiesbaden. https://doi.org/10.1007/978-3-662-55743-3.

Wang, X., Li, X., Shen, Z., Ma, Y., Liu, H. (2018), "Finite element simulation on investigations, modeling, and multiobjective optimization for clinch joining process design accounting for process parameters and design constraints", The International Journal of Advanced Manufacturing Technology, Vol. 96, pp. 3481-3501. https://doi.org/10.1007/s00170-018-1708-4.

Witten, I. H., Eibe, F., Hall, M. A., Pal, C. J. (2017), Data Mining. Practical Machine Learning Tools and Techniques, Morgan Kaufmann, Cambridge.

Zirngibl, C., Schleich, B. and Wartzack, S. (2020), “Potentiale datengestützter Methoden zur Gestaltung und Optimierung mechanischer Fügeverbindungen”, Proceedings of the 31. Symposium Design for X, online, 16.-17.09.2020, Krause, D.; Paetzold, K.; Wartzack, S., pp. 71-80. https://doi.org/10.35199/dfx2020.8. 